\title{
Modelling Collision Potentials in Port Anchorages: Application of the Navigational Traffic Conflict Technique (NTCT)
}

\author{
Ashim Kumar Debnath ${ }^{1}$ and Hoong Chor Chin ${ }^{2}$ \\ ${ }^{1}$ (Centre for Accident Research and Road Safety - Queensland (CARRS-Q), \\ Queensland University of Technology, Australia) \\ ${ }^{2}$ (National University of Singapore, Singapore) \\ (E-mail: ashim.debnath@qut.edu.au)
}

\begin{abstract}
Despite the extent of works done on modelling port water collisions, not much research effort has been devoted to modelling collisions at port anchorages. This paper aims to fill this important gap in the literature by applying the Navigation Traffic Conflict Technique (NTCT) to measuring the collision potentials in anchorages and for examining the factors contributing to collisions. Building on the principles of the NTCT, a collision potential measurement model and a collision potential prediction model were developed. These models were illustrated by using vessel movement data of the anchorages in Singapore port waters. Results showed that the measured collision potentials are in close agreement with those perceived by harbour pilots. Higher collision potentials were found in anchorages attached to the shoreline and international fairways, but not at those attached to confined water. Higher operating speeds, larger numbers of isolated danger marks and day conditions were associated with reduction in the collision potentials.
\end{abstract}

\author{
KEYWORDS \\ 1. Port safety. $\quad$ 2. Ship collision. $\quad 3$. Singapore port.
}

Submitted: 9 November 2014. Accepted: 22 June 2015. First published online: 10 July 2015.

1. INTRODUCTION. Collisions in port waters account for a major share of the all port-water accidents involving vessels (Goossens and Glansdorp, 1998; Akten, 2004; Darbra and Casal, 2004; Yip, 2008). A number of researchers (e.g., Debnath and Chin, 2007; Chin and Debnath, 2008; Chin et al., 2010; Debnath and Chin, 2010; Weng et al., 2012) have contended that navigational collisions would remain a major concern for many seaports due to a rapid increase in the numbers and sizes of vessels in many navigational areas (Soares and Teixeira, 2001).

To address this important safety concern in port water navigation, many researchers (e.g., Goossens and Glansdorp, 1998; Akten, 2004; Darbra and Casal, 2004; Liu et al., 
2006; Yip, 2008) have looked at understanding the characteristics and causes of collisions by analysing the historical records of navigational accidents. As an alternative to the historical data, recently some researchers (e.g., Debnath and Chin, 2006; Debnath and Chin, 2010; Montewka et al., 2010; Debnath et al., 2011; Weng et al., 2012; Li et al., 2014) have looked at surrogate indicators of the collision events, such as navigational traffic conflicts and close encounters between vessels.

Apart from the efforts on the analysis of historical collision records and surrogate indicators of collision events, researchers looked at the safety issues related to navigational collisions using a variety of methodological approaches, such as traffic flow-based methods (Montewka et al., 2012; Silveira et al., 2013), traffic simulation-based methods (van Dorp and Merrick, 2011; Blokus-Roszkowska and Smolarek, 2012; Goerlandt et al., 2012), methods using traffic indices (Qu et al., 2011; Suman et al., 2012), Bayesian networks (Hänninen et al., 2013; Montewka et al., 2014), and fuzzy failure mode and effect analysis methods (Zaman et al., 2014). The IALA IWrap MKII model (IALA, 2012) presented guidelines for estimating collision frequencies by multiplying the number of collision candidates with causation probability. Burmeister et al. (2014) provided a detailed description of the IWrap model in the context of collisions in anchorages. Goerlandt and Kujala (2014) reviewed the different methodological approaches for studying ship-ship collisions and found a low inter-method reliability among the approaches, thus highlighting the need for further research on refining the existing methods or developing new methods. Sormunen et al. (2014) further discussed the uncertainty of using traffic simulation-based methods and highlighted that simulation methods should be improved for estimating how ship encounter scenarios develop into collision scenarios. Arguably, using real-world traffic movement data for understanding how the encounter scenarios develop into collision scenarios might help in reducing the uncertainty present in traffic simulation models.

A common methodological approach of utilising real-world traffic movement data is the surrogate indicators-based method. The safety analysis approach based on the surrogate indicators of collisions is appealing, because it overcomes the major limitations of the traditional approach that relies on historical collision records. Debnath and Chin (2010) have discussed this issue in detail in their article which introduced a novel and proactive approach for measuring the risks of collisions, namely the Navigational Traffic Conflict Technique (NTCT). The NTCT utilises traffic conflicts as an alternative to historical collision records, thus it allows managing collisions in a proactive manner. The major benefit of using NTCT is that safety analysts do not need to wait for years so that a large number of collisions can be accrued - accumulating a large number of collisions might actually not be possible since these collisions are random and sporadic events, and are subjected to continuous changes in waterway conditions and navigational aidsrather preventive or corrective actions can be taken by analysing traffic movements data from a relatively short period of time.

Despite the recent developments in the area of developing proactive safety management techniques, little research has been carried out in applying these techniques for port anchorages. Almost all research efforts (e.g., Debnath and Chin, 2010; Montewka et al., 2010; Debnath et al., 2011; Weng et al., 2012) have been devoted so far to examining safety issues related to collisions in fairways and channels. A major part of vessel movements within port waters occur in fairways and channels, which might be why these waters have been a target of considerable research. 
Collision statistics from port waters indicate that the likelihood of collision could be high in other parts of port waters, particularly in anchorages. For example, Yip (2008) reported from an analysis of navigational accidents in Hong Kong waters that $20 \%$ of collisions occur in anchorages (some of which might include barge traffic), whereas only $10 \%$ occur in fairways. Moreover, Liu et al. (2006) argued that often vessels anchor arbitrarily in busy seaports. In particular, vessels with a clear departure schedule tend to anchor near boundaries of channels or fairways for easy access. This arbitrary anchoring practice, in combination with a high density of vessels (both anchored and underway) could result in having limited space between vessels, thus creating difficult manoeuvring processes for vessels intending to anchor or to come out of anchorages (Usui, 2002). Consequently, these factors might lead to collisions involving moving vessels and anchored vessels. Burmeister et al. (2014) argued that anchorages pose risk to navigational safety as anchored vessels might act as obstacles leading to collisions with moving vessels. Proper positioning of anchored vessels has been highlighted as an important technique for avoiding collisions with moving vessels by Zhang and Zhao (2013) who also argued that such collisions are not uncommon in port waters. In summary, relatively little attention has been given to understand the safety issues related to anchorages in port waters, compared to other port waters (e.g., fairways). In a recent study, Burmeister et al. (2014) studied collision risks in anchorages using the IALA IWrap MkII model (IALA, 2012). They presented a methodology for extending the use of IWrap model in assessing the frequency and material consequences of collisions between an anchored vessel and a vessel underway. The developed methodology was illustrated using simulated data. While this study marks as a good attempt to estimate collision frequencies in anchorages, further research is required, particularly on 1) using real-world traffic movement data instead of simulated data, and 2) examining the contributing factors of collisions in anchorages.

This paper aims to fill these important gaps in port navigational safety literature by applying the NTCT for measuring collision potentials in anchorages using real-world traffic movement data and for examining the factors contributing to the collisions. Specifically, a proactive Collision Potential Measurement model is developed which provides a quantitative measurement of the collision potentials in anchorages. For examining the effects of contributing factors, a prediction model is developed which examines the relationships between the collision potentials and the geometric, traffic, and regulatory control characteristics of anchorages. Both the measurement and prediction models are illustrated for anchorages within Singapore port waters by using vessel movement data obtained from the Port's Vessel Traffic Information System (VTIS). The rest of the paper discusses the two models first, followed by a description of the data used for illustrating the models. Model calibration and validation results are presented next, before providing concluding remarks.

\section{METHOD}

2.1. Collision Potential Measurement Model (CPMM). The NTCT was developed by Debnath and Chin (2010) and looks at surrogate indicators of navigational collisions for estimating the collision potentials in waterways. The fundamental principle behind the NTCT methodology is based on the hypothesis that all interactions between vessels fall within a continuum of safety related events (Debnath and Chin, 2006) with collisions at the top of a hierearchical system. A key feature is that 
"close proximity" vessel interactions (also known as "collision candidates") - situations in which two or more vessels are sufficiently close in space and time and whose trajectories will cross unless evasive actions are taken - reside just below collisions in the hierarchy. These interactions are called near-misses, traffic conflicts, or surrogate measures of collision potential. NTCT focuses on these surrogate indicators of collision events in order to derive a quantitative estimate of the probability of collision in a two-vessel interaction or collectively for all interactions within a waterway (i.e., fairway, anchorages).

To estimate the collision potential in an anchorage for a given time period, at first it is necessary to estimate the collision potentials for all two-vessel interactions within the anchorage. Navigational traffic movement is essentially two-dimensional in nature (particularly within anchorage waters where vessels need to navigate through the spaces available between anchored vessels). Therefore, the closeness of two vessels to a potential collision event should be represented both in terms of spatial and temporal closeness. The Distance at Closet Point of Approach (DCPA) and Time to Closest Point of Approach (TCPA) indicators were proposed by Debnath and Chin (2010) for representing the spatial and temporal closeness between a pair of vessels. These indicators are widely used in on board navigation and navigational research and thus have general acceptability to navigators and researchers. Research (e.g., Chin and Debnath, 2009; Debnath and Chin, 2010) showed that these indicators are well capable of representing the collision potential of an interaction.

To derive the collision potential in a vessel interaction as a function of the DCPA and TCPA, Chin and Debnath (2009) developed a set of statistical regression models for different vessel size classes. By employing these models, the collision potential in an interaction can be estimated at short intervals (e.g., 2-5 seconds) throughout the entire interaction process. It should be noted that interactions in which vessels are far away from each other (i.e., a collision is not probable) need to be excluded from this estimation process. The concept of Ship Domain (SD) was proposed as an acceptable criterion for defining which vessels to exclude (see Debnath (2009) for details). Therefore, the collision potentials were estimated for the interactions where one vessel was within the SD of a vessel that is present in an anchorage (these interactions are termed as 'encounters' hereafter). Each vessel in an anchorage was paired with all other vessels within the SD of the vessel in the anchorage and the collision potential for each pair was estimated.

From these estimated collision potentials at different timestamps during an encounter process involving a pair of vessels, the most severe point of the encounter (when the collision potential is the highest) can be identified. Let us take $C_{\max }$ as the highest collision potential found in an interaction. Converting $C_{\max }$ to $C_{\max }^{\prime}=\left(1 /\left(1-C_{\max }\right)\right)$ and considering all $C_{\max }^{\prime}$ values within an anchorage, a left-truncated distribution (at $\left.C_{\text {max }}^{\prime}=1\right)$ with an asymptotic tail towards right can be obtained. Since $C_{\max }^{\prime}$ represents the severity of a conflict in terms of closeness to a collision event (i.e., a higher value indicates more likelihood of collision), the tail of the curve could be utilised to estimate the collision potential in an anchorage by setting a threshold value that will separate the serious conflicts from the non-serious ones. A serious conflict corresponds to vessel encounter that may lead to a certain collision if appropriate evasive actions are not taken urgently. Empirical results from a study (Debnath, 2009), which tested a set of left-truncated distributions to obtain the best-fit distribution, showed that a 
truncated gamma distribution consistently provides the best fit for the distribution of $C_{\max }^{\prime}$ values. The cumulative distribution function of the truncated gamma distribution can be written as:

$$
F_{C_{\max }^{\prime}}\left(C_{\max }^{\prime}\right)=p(0)+\left[1-p(0) \int_{\theta}^{\tau} \frac{1}{\Gamma \gamma \times \delta^{\gamma}}(q-\theta)^{\gamma-1} \exp \frac{-(q-\theta)}{\delta} d q\right]
$$

where $p(0)$ is a probability mass function which represents the proportions of nonconflict encounters (when two vessels are not likely to collide); $\tau$ is a threshold value which distinguishes the serious conflicts from the non-serious ones; $\gamma$ and $\delta$ are the estimated shape and scale parameters of the gamma distribution respectively; and $\theta$ is the threshold parameter representing the truncation value $(=1)$.

Since the collision potentials vary with vessel sizes, it is necessary to consider different threshold values $(\tau)$ for different vessel classes (vc). Considering $p_{v c}$ as a probability mass function of different vessel classes, the collision potential in an anchorage can be expressed as:

$$
C P=p\left(C_{\max }^{\prime}>\tau_{v c}\right)=\sum_{v c=1}^{M}\left[1-F_{\tau_{v c}}\left(\tau_{v c}\right)\right] \times p_{v c}
$$

where $\tau_{v c}$ is the threshold value for vessel class $v c$; and $\mathrm{M}$ is the total number of vessel classes.

The collision potential $(C P)$ expresses the overall probability of collision in an anchorage in terms of the probability of serious conflict per vessel encounter. Types of collisions in anchorages could be broadly classified into three categories based on the types of vessels involved: 1) collisions between two anchored vessels (including drifting cases), 2) collisions between an anchored vessel and a vessel underway, and 3) collisions between two vessels underway, as defined by Burmeister et al. (2014). Since the current study analysed collision potentials of all possible vessel pairs including the anchored vessels and the vessels underway (i.e., all vessels within the SD of a particular vessel in an anchorage), the estimated collision potential in the anchorage was an aggregated value for all collision categories. Separate analyses for each category of collisions were not performed in the current study.

To capture the effects of visibility and presence of navigational aids, the collision potentials should be measured separately for day and night periods.

2.2. Collision Potential Prediction Model (CPPM). While the CPMM measures the collision potentials in anchorages by analysing the vessel movement trajectories in the anchorages, the CPPM allows predicting the collision potentials by inputting known characteristics of the anchorages in the CPPM. The CPPM establishes relationships between the collision potentials (estimated by the CPMM) and the geometric, traffic, and regulatory control characteristics of anchorages. It models the $C P$ values in each encounter as a dichotomous response variable: serious conflict $(=1)$ and non-serious conflict $(=0)$. Grounding on the modelling framework developed by Debnath et al. (2011), this response variable can effectively be modelled as proportional data (i.e., proportions of serious conflicts among all encounters). A Binomial Logistic Model (BLM) is an ideal choice for modelling such proportional data. 


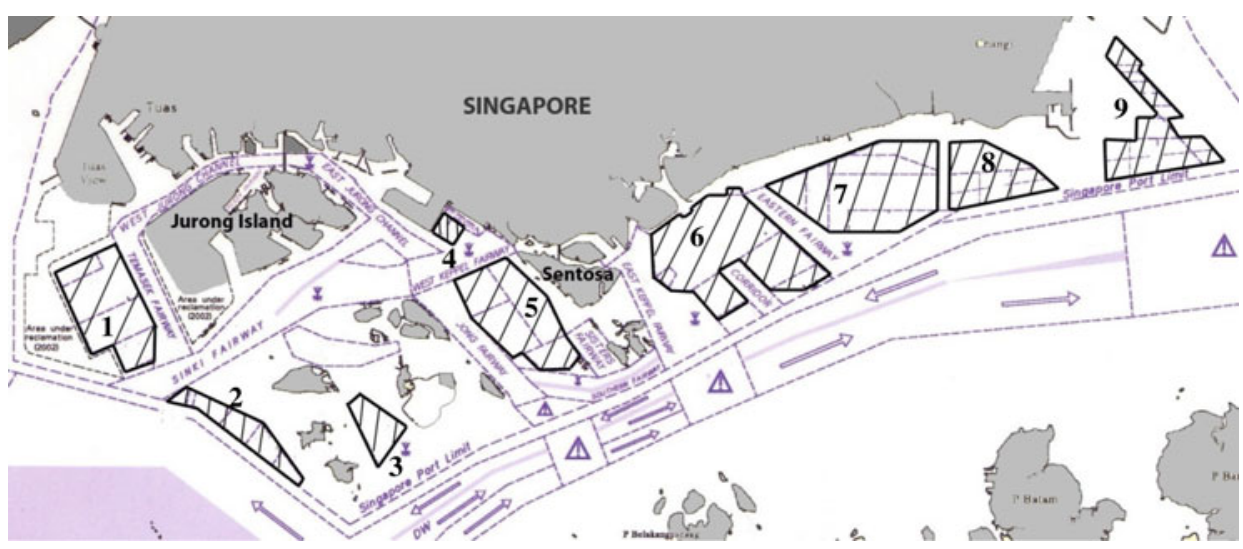

Figure 1. Anchorages in Singapore port waters (numerically numbered and marked as hatched areas).

The BLM expresses the occurrence probability of a serious conflict $\left(p_{\text {eat }}\right)$ for an encounter $e$ at time $t$ in an anchorage $a$ as:

$$
p_{\text {eat }}=E\left(\frac{S C_{a t}}{S C_{a t}+N S C_{a t}}\right)=\frac{\exp \left(\boldsymbol{\beta} \mathbf{X}_{\text {eat }}\right)}{1+\exp \left(\boldsymbol{\beta} \mathbf{X}_{\text {eat }}\right)}
$$

where $\mathbf{X}_{\text {eat }}$ is a vector of explanatory variables representing the geometric, traffic, and regulatory control characteristics of anchorage $a ; \boldsymbol{\beta}$ is a vector of regression coefficients; and $S C_{a t}$ and $N S C_{a t}$ are the numbers of serious conflicts and non-serious conflicts, respectively, in anchorage $a$ at time $t$.

3. DATA. The CPMM and CPPM are illustrated by using vessel movement data of the anchorages in Singapore port waters (see Figure 1 for a map which shows nine clusters of anchorages). This data, obtained from the VTIS of the Port, include vessels' trajectories (position coordinates, speeds, headings) and numeric identities. The data fields are generally updated at short intervals (a few seconds) depending on the speeds and density of vessels at the anchorages. Four hours' data from each of day and night periods were used in the illustrative example. Based on the results from existing studies (Debnath and Chin, 2010; Debnath et al., 2011), four hours' data was deemed sufficient for obtaining statistically reliable results.

A total of 15 explanatory variables, which are hypothesized to relate to the collision potentials in anchorages, were included in the CPPM. The variables were selected based on the existing knowledge from the literature (e.g., Debnath et al., 2011; Debnath and Chin, 2009b), local knowledge regarding the anchorages in the study area and availability of information related to the geometric and traffic characteristics of the anchorages. Among these variables, four were excluded from the model due to multi-collinearity. The definitions of the remaining variables, together with their means and standard deviations (S.D.), are presented in Table 1.

Since collision potentials in anchorages are likely to be influenced by traffic in its boundary waters, it is necessary to consider the boundary effects in the CPPM. 
Table 1. Explanatory variables included in the RPM.

\begin{tabular}{llrr}
\hline Explanatory variables & Description & Mean & S.D. \\
\hline Anchorage characteristics & & & \\
$\quad$ Anchorage boundary & & $0 \cdot 667$ & $0 \cdot 485$ \\
$\quad$ Shoreline & 1 if present, else 0 & $0 \cdot 667$ & $0 \cdot 485$ \\
$\quad$ Intersection & 1 if present, else 0 & $0 \cdot 333$ & $0 \cdot 485$ \\
Confined water & 1 if present, else 0 & $0 \cdot 667$ & $0 \cdot 485$ \\
$\quad$ International fairway & 1 if present, else 0 & $16 \cdot 389$ & $4 \cdot 164$ \\
Water depth & Controlling water depth of navigation (meters) & $0 \cdot 333$ & $0 \cdot 970$ \\
Cardinal mark & Number of cardinal marks & $0 \cdot 333$ & $0 \cdot 485$ \\
Isolated danger mark & Number of isolated danger marks & $1 \cdot 194$ & $0 \cdot 818$ \\
Traffic characteristics & & $2 \cdot 693$ & $2 \cdot 257$ \\
$\quad$ Dynamic ship density & Avg. dynamic ship density in anchorage (ships/sq NM) & $2 \cdot 419$ & $2 \cdot 032$ \\
Stationary ship density & Avg. stationary ship density in anchorage (ships/sq NM) & & \\
$\quad$ Operating speed & Average operating speed in anchorage (knots) & $0 \cdot 500$ & $0 \cdot 514$ \\
Time variable & 1 if night, 0 if day & & \\
Day/Night & &
\end{tabular}

Therefore, the waters around an anchorage were described in five categories: shoreline, intersection, confined water, local fairway, and international fairway. These categories were treated as dichotomous variables in the model based on their presence. Since local fairways and confined water were highly correlated, only the confined water variable was included in the model. The port terminal berth areas and the low depth waters with scattered land obstacles were defined as confined waters. The fairways inside port waters were termed as local fairways, whereas those outside port waters were the international fairways.

Geometric characteristics of anchorages included the controlling water depth of navigation, presence of pilot boarding/disembarkation areas and the ratio of area to perimeter of anchorage. Pilot boarding/disembarkation areas were defined as the waters used by pilots to board or disembark an ocean-going vessel. The area-perimeter ratio was preliminarily considered to examine if there were any effects of the anchorage shape on collision potential, but it was omitted due to multi-collinearity. In addition, the variable representing presence of pilot boarding/disembarkation area was omitted from the analysis.

Characteristics of navigational aids (e.g., navigational buoys/lights) were represented by cardinal marks, isolated danger marks and safe water marks, as specified in the IALA Maritime Buoyage System (IALA, 1980). A cardinal mark indicates the safe side of the mark which shall be passed by vessels, whereas an isolated danger mark is used to indicate dangers (e.g., a small low-depth area which is surrounded by navigable waters). The safe water mark variable was omitted due to multi-collinearity.

Traffic characteristics included the average density of dynamic ships per square nautical mile, the average density of stationary ships per square nautical mile and the mean operating speed in an anchorage. A binary variable indicating day and night periods was considered to represent the navigational characteristics at these time periods.

\section{ANALYSIS RESULTS}

4.1. Measured Collision Potentials in anchorages. Collision potentials were measured by classifying vessels into four groups (based on vessel sizes): category 1 had 
Table 2. Collision potentials measured by CPMM and perceived by harbour pilots.

\begin{tabular}{|c|c|c|c|c|c|c|c|c|}
\hline \multirow{3}{*}{$\begin{array}{l}\text { Anchorage } \\
\text { No. }\end{array}$} & \multicolumn{4}{|c|}{ Collision potential measured by $\mathrm{CPMM}^{\wedge \wedge}$} & \multicolumn{4}{|c|}{ 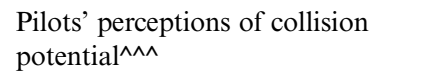 } \\
\hline & & Day & & Night & & Day & & Night \\
\hline & Day & $\operatorname{Rank}^{\wedge}$ & Night & $\operatorname{Rank}^{\wedge}$ & Day & $\operatorname{Rank}^{\wedge}$ & Night & Rank $^{\wedge}$ \\
\hline 1 & $1 \cdot 060 \mathrm{E}-02$ & 1 & 8.932E-03 & 6 & $38 \cdot 89$ & 1 & 38.89 & 5 \\
\hline 2 & $2 \cdot 102 \mathrm{E}-04$ & 6 & 1.011E-02 & 5 & $5 \cdot 88$ & 7 & $29 \cdot 41$ & 7 \\
\hline 3 & 7.995E-08 & 9 & 1.051E-05 & 9 & $0 \cdot 00$ & 8 & $17 \cdot 65$ & 9 \\
\hline 4 & $6 \cdot 844 \mathrm{E}-04$ & 5 & $1 \cdot 260 \mathrm{E}-02$ & 4 & $17 \cdot 65$ & 3 & $52 \cdot 94$ & 3 \\
\hline 5 & 8.893E-03 & 2 & 3.046E-02 & 1 & $23 \cdot 53$ & 2 & $72 \cdot 22$ & 1 \\
\hline 6 & $3 \cdot 718 \mathrm{E}-03$ & 4 & 1.594E-02 & 3 & $16 \cdot 67$ & 5 & $41 \cdot 18$ & 4 \\
\hline 7 & $7 \cdot 438 \mathrm{E}-03$ & 3 & $2 \cdot 157 \mathrm{E}-02$ & 2 & $17 \cdot 65$ & 4 & $58 \cdot 82$ & 2 \\
\hline 8 & $6 \cdot 759 \mathrm{E}-05$ & 7 & $8 \cdot 596 \mathrm{E}-03$ & 8 & $0 \cdot 00$ & 8 & $27 \cdot 78$ & 8 \\
\hline 9 & $3 \cdot 862 \mathrm{E}-06$ & 8 & $8 \cdot 621 \mathrm{E}-03$ & 7 & $11 \cdot 11$ & 6 & 38.89 & 5 \\
\hline
\end{tabular}

$\wedge^{\wedge}$ Values ranked in descending order, ${ }^{\wedge \wedge}$ Represent the probability of a serious conflict per encounter; ${ }^{\wedge \wedge \wedge ~} \%$ pilots gave a rating of 3 or 4 on a scale of 0 to 4 (0: very unlikely, 1: Unlikely, 2: Moderate chance, 3: Likely, and 4: Very likely)

gross tonnage (GT) between 300 and 12,000; category 2 had GT between 12,001 and 20,000; category 3 had GT between 20,001 and 75,000; and category 4 had GT more than 75,000 . For each of these categories, the threshold values for separating serious conflicts from non-serious ones were adopted from Debnath and Chin (2010).

The measured collision potentials (i.e., probability of a serious conflict per encounter) for the anchorages studied are presented in Table 2 . The probability values range from 8 in 100,000,000 to 1 in 100 during the day hours, and 1 in 100,000 to 3 in 100 during the night hours. The finding that the collision potentials are higher during the night hours than the day hours is consistent with findings obtained in earlier studies (e.g., Chin and Debnath, 2009; Debnath et al., 2011).

To validate the CPMM, the measured collision potentials of the anchorages were compared with those perceived by harbour pilots for the same anchorages. Existing research (Debnath and Chin, 2009a) showed that harbour pilots have a reasonably good understanding of the actual probabilities of collision in port waters. The perceived values were obtained from Debnath and Chin (2009a) in which a detailed description of the perception survey can be found. In the survey, pilots were asked to rate the collision potentials of the anchorages shown in Figure 1. A five point scale representing the likelihood of a serious conflict in an anchorage (0: Very unlikely, 1: Unlikely, 2: Moderate chance, 3: Likely, and 4: Very likely) was used to collect the perceived ratings. From a pilot testing of the survey among several experienced pilots, it was decided to use the term 'close quarter situation', instead of 'serious conflict' in the survey as pilots are more familiar with the former term and both terms essentially carry a similar meaning. Pilots were asked to provide the ratings in each anchorage for an average pilotage job with an average vessel size that they generally operate (it is to be noted that Singapore port pilots generally operate vessels of one of the four size categories outlined in the previous paragraph, based on their experience and rank). The ratings were obtained separately for day and night periods. The survey, participation in which was voluntary and responses in which were anonymous, was sent to 160 harbour pilots of the Port of Singapore. A total of 70 completed survey forms ( $44 \%$ 


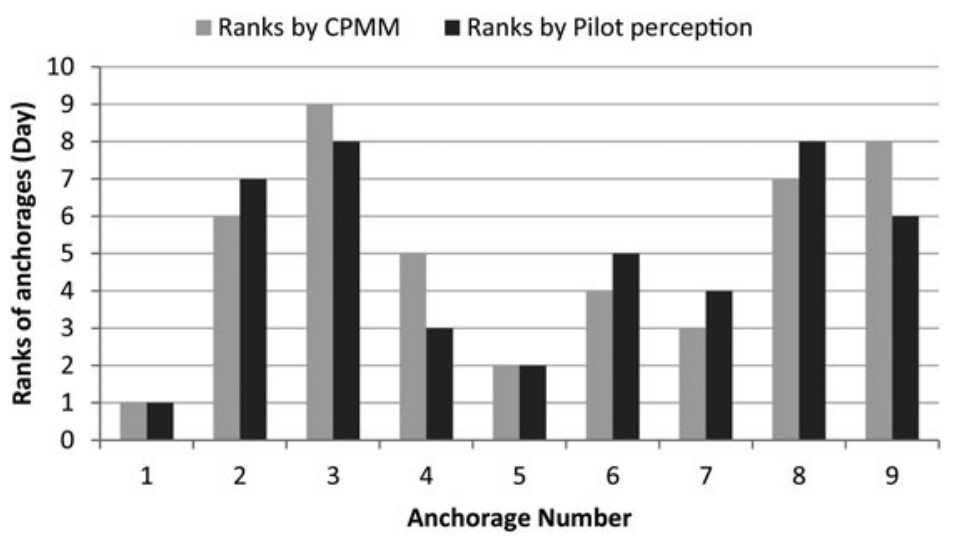

Figure 2. Comparison of the anchorage rankings for Day period.

response rate) were received. The age of respondents ranged from 28 to 61 years with a mean and standard deviation of 43.0 years and $9 \cdot 8$ years respectively. The respondents had an average 11.3 years' experience as harbour pilots with a standard deviation of $10 \cdot 9$ years.

The anchorages are ranked in descending order of collision potentials for both the measured (by using the values obtained from the CPMM) and the perceived values (by computing the percentage of pilots giving a score of 3 or 4 on the five point scale). Comparison of the rankings for the day and night periods (presented in Figure 2 and Figure 3 respectively) indicate that the rankings of the anchorages according to the collision potentials measured by the CPMM match the rankings by the pilot perceptions reasonably well.

4.2. Regression Model Results. The parameters of the BLM were derived using the maximum likelihood estimation method. The potential correlations among observations within an anchorage were modelled using a modified sandwich variance matrix approach (see Debnath (2009) for a detailed description of this approach). Starting with a saturated model that included all of the explanatory variables, a backward elimination procedure was employed to obtain the most parsimonious model by minimising the value of Akaike Information Criteria (AIC). The insignificant variables were omitted one after another starting with the most insignificant one. Estimates of the BLM along with their fitness statistics are presented in Table 3.

The most parsimonious BLM had an AIC value of 63.9 and a dispersion statistic of 0.83 (so adjustments to standard errors are not required). The Likelihood Ratio statistic of the model was $231 \cdot 3$, which is well above the critical value at $99 \%$ confidence level, implying that the model has a reasonably good fit. The Adjusted Log-likelihood Ratio Index $(=0 \cdot 77)$ also indicates that the model has sufficient explanatory and predictive power.

Turning to specific estimation results, the collision potential is significantly associated with presence of shoreline at the anchorage boundary (beta $=5.52, p<0.001$ ). Anchorages attached to the shoreline have 247 times higher odds of a serious conflict. Vessels have restricted access to this type of anchorage due to the presence of a shoreline at a boundary of these anchorages. Typically, vessels anchored near the shoreline need to navigate through the other anchored vessels in order to move out of the 


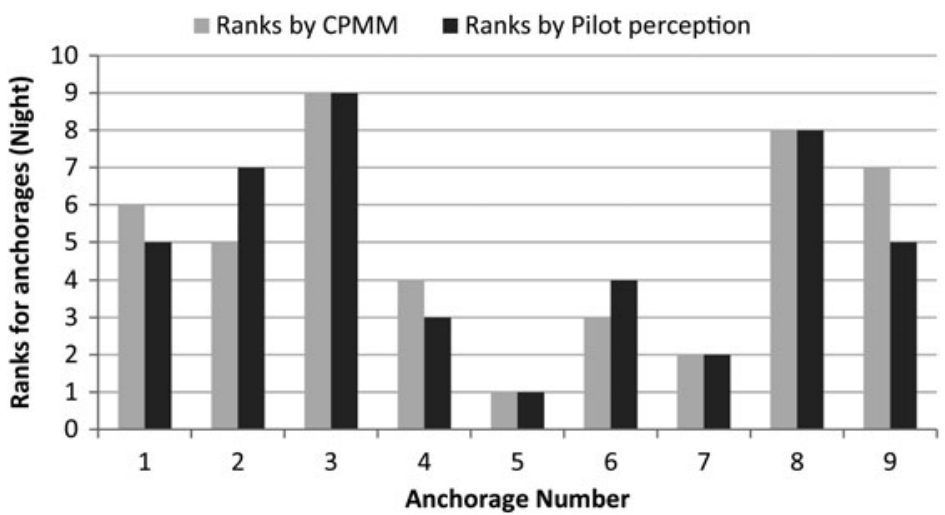

Figure 3. Comparison of the anchorage rankings for Night period.

Table 3. Regression estimates of the RPM.

\begin{tabular}{|c|c|c|c|c|c|}
\hline \multirow[b]{2}{*}{ Explanatory variables } & \multicolumn{2}{|c|}{ Effect estimates } & \multirow[b]{2}{*}{ Odds ratio } & \multirow[b]{2}{*}{ Z-stat } & \multirow[b]{2}{*}{ P-value } \\
\hline & Coefficient & S.E. & & & \\
\hline \multicolumn{6}{|l|}{ Anchorage characteristics } \\
\hline \multicolumn{6}{|l|}{ Anchorage boundary } \\
\hline Shoreline & $5 \cdot 5156$ & $0 \cdot 4307$ & $248 \cdot 543$ & $12 \cdot 80$ & $0 \cdot 000$ \\
\hline Confined water & -5.5356 & $0 \cdot 4768$ & $0 \cdot 004$ & $-11 \cdot 61$ & $0 \cdot 000$ \\
\hline International fairway & $3 \cdot 8023$ & $0 \cdot 4997$ & $44 \cdot 803$ & $7 \cdot 61$ & $0 \cdot 000$ \\
\hline Isolated danger mark & $-4 \cdot 3017$ & $0 \cdot 6901$ & $0 \cdot 014$ & $-6 \cdot 23$ & $0 \cdot 000$ \\
\hline \multicolumn{6}{|l|}{ Traffic characteristics } \\
\hline Operating speed & $-0 \cdot 4991$ & $0 \cdot 1689$ & 0.607 & $-2 \cdot 95$ & 0.003 \\
\hline \multicolumn{6}{|l|}{ Time variable } \\
\hline Day/Night & $2 \cdot 0819$ & $0 \cdot 8520$ & 8.020 & $2 \cdot 44$ & $0 \cdot 015$ \\
\hline \multicolumn{6}{|l|}{ Model statistics } \\
\hline Intercept & $-9 \cdot 8153$ & 0.6148 & & -15.96 & $0 \cdot 000$ \\
\hline Log-likelihood (null) & $-140 \cdot 621$ & & & & \\
\hline Log-likelihood (model) & $-24 \cdot 962$ & & & & \\
\hline Likelihood ratio statistics & $231 \cdot 318$ & & & & \\
\hline Adj. LL ratio index & 0.773 & & & & \\
\hline AIC & $63 \cdot 924$ & & & & \\
\hline Dispersion parameter & $0 \cdot 825$ & & & & \\
\hline
\end{tabular}

anchorage. Such movements generate more interactions (and possibly more conflicts) between vessels, resulting in higher probabilities of collision.

Anchorages bounded by confined waters have lower collision potentials (beta $=-5 \cdot 54$, $\mathrm{p}<0.001)$ with 250 times higher odds of non-serious conflicts. Confined water characterises low density and slow speed vessel movements in the berth areas, and only small vessels (e.g., pilot boats, speed boats) operate in these low depth waters. Collectively, these factors might reduce the collision potentials in anchorages bounded by confined waters.

The probability of serious conflicts significantly increases if an international fairway is present at the anchorage boundary (beta $=3 \cdot 80, \mathrm{p}<0 \cdot 001$ ). Pilot boarding/disembarkation areas are usually located near the international fairways. These areas are 
used by pilots to go on board the vessels calling to a port or to disembark the vessels intending to leave the port. The boarding and disembarkation process is a safety critical event in navigation (SOLAS, 1974) and it often requires vessels to reduce speeds to make the process safer. Such reduction in speed could impede through-traffic movements in international fairways and, possibly, result in a greater number of conflicts because of the variation in speeds among successive vessels. In addition, interactions of pilot boats with the existing traffic may pose an additional likelihood of collision. Results showed that the odds of a serious conflict are 44 times higher if an international fairway is present at an anchorage boundary.

The number of isolated danger marks in anchorages have significant negative association with collision potentials in anchorages (beta $=-4.30, \mathrm{p}<0.001$ ). An isolated danger mark decreases the odds of a serious conflict by $98 \cdot 6 \%$. However, other research found that presence of an isolated danger mark increases the odds of serious conflicts in fairways (Debnath et al., 2011). The difference in the effects of isolated danger marks in fairways and anchorages could be observed due to the fact that operating speed is generally higher in fairways than in anchorages. At high speeds, it is necessary to take collision avoidance actions at an early stage. Failing to do so may increase the probability of collision. On the other hand, vessels operate at lower speeds in anchorages, thus it is possible to plan collision avoidance actions early.

Operating speed showed significant negative association with collision potentials in anchorages. An increase of 1 knot reduced the odds of a serious conflict by $39 \cdot 3 \%$ (beta $=-0 \cdot 50, p=0 \cdot 003$ ). This negative association might have been observed for a couple of reasons. Pilots might navigate at lower speeds when they foresee a difficult navigational situation ahead, arising from high density of anchored ships (i.e., less navigation room available than in a low density condition) or presence of other moving vessels nearby. The track-keeping ability of vessels, which is reduced at lower speeds, might also contribute to this negative association.

Pilots might navigate at higher speeds when the density of anchored ships is low (i.e., there is more navigation room and possibly less likelihood of collision) and vice versa. The track-keeping ability of vessels, which is reduced at lower speeds, might also contribute to this negative association.

The collision potentials were found to be higher during the night hours (beta $=2 \cdot 08$, $p=0 \cdot 015$ ) with $7 \cdot 0$ times higher odds of a serious conflict than during the day hours. This finding is consistent with those of other studies (Chin and Debnath, 2009; Debnath et al., 2011; Weng et al., 2012). Arguably, the speeds and distances between vessels and even any moderate changes in course can be more readily judged during day than during night. At night, pilots need to rely on navigational aids (e.g., radar, navigational lights), which makes the risk perception and mitigation process more difficult than in the daytime. Effectiveness of navigational lights can also be reduced at night due to bright background lights on shore and from nearby islands (Akten, 2004; Liu et al., 2006). A number of studies (Chin and Debnath, 2009; Debnath and Chin, 2009a; Debnath and Chin, 2009b) have also reported that pilots perceive higher collision risks at night.

5. CONCLUSIONS. Significant research efforts have been devoted to examining the safety issues related to collisions in port fairways and channels, but little attention has been given to understanding the safety issues at port anchorages. This paper aimed 
to fill this important gap in the literature by measuring collision potentials in anchorages and establishing relationships between the collision potentials and the various geometric, traffic, and regulatory control characteristics of anchorages.

A collision potential measurement model was developed by using the principles of the NTCT. This model provided a quantitative estimate of collision potentials in anchorages by analysing surrogate indicators of collision events (i.e., traffic conflicts). Collision potentials were expressed in terms of the probability of a serious conflict (an encounter which may lead to a collision event if appropriate evasive actions are not taken urgently) per vessel encounter (an interaction event between two vessels when one is within the ship domain of the others). The probabilities of serious conflicts were later modelled in a BLM framework to derive a prediction model. This model estimated the relationships between the probabilities of serious conflicts and the geometric, traffic, and regulatory control characteristics of anchorages.

Both the measurement and prediction models were illustrated for the anchorages in Singapore port waters. Results showed that the estimated collision potentials match those perceived by harbour pilots reasonably well thus providing evidence for the validity of the measurement model. Estimation results of the BLM showed that the collision potentials are higher in anchorages attached to shoreline and international fairways. On the other hand, the anchorages bounded by confined water had lower collision potentials. Similarly, lower collision potentials were observed in anchorages with lower operating speeds and higher numbers of isolated danger marks. Overall, the collision potentials in night hours were higher than those in day hours.

Future research should focus on 1) improving the CPMM by incorporating information related to vessel command (alertness or competence) into the information related to vessel movement trajectories, 2) expanding the CPPM by including more explanatory variables related to the geometric and traffic characteristics of the anchorages (e.g., the number of passing traffic on the fairways adjacent to the anchorages, types of vessels involved), and 3) extending the analyses of collision potentials in the anchorages by considering the different types of vessel interactions (e.g., interactions among anchored-anchored, anchored-underway, and underway-underway vessels).

\section{REFERENCES}

Akten, N. (2004). Analysis of Shipping Casualties in the Bosphorus. The Journal of Navigation, 57(03), $345-356$.

Blokus-Roszkowska, A. and Smolarek, L. (2012). Collision risk estimation for motorways of the sea. Reliability: Theory \& Applications, 2(25), 58-68.

Burmeister, H.-C., Walther, L., Jahn, C., Tãster, S. and Froese, J. (2014). Assessing the Frequency and Material Consequences of Collisions with Vessels Lying at an Anchorage in Line with IALA iWrap MkII. TransNav, the International Journal on Marine Navigation and Safety of Sea Transportation, 8(1), 61-68.

Chin, H.C. and Debnath, A.K. (2008). Statisical Analysis of Conflict Involvements in Port Water Navigation. Proceedings of the MARTECH 2008, Singapore Polytechnic, Singapore.

Chin, H.C. and Debnath, A.K. (2009). Modeling perceived collision risk in port water navigation. Safety Science, 47(10), 1410-1416.

Chin, H.C., Debnath, A.K. and Wang, Y. Y. (2010). Proactive Management of Collision Risks in Port Waters using Navigational Traffic Conflicts. MARTECH 2010. Singapore.

Darbra, R.-M. and Casal, J. (2004). Historical analysis of accidents in seaports. Safety Science 42(2), 85-98.

Debnath, A.K. (2009). Traffic-conflict-based modeling of collision risk in port waters. Doctoral thesis, National University of Singapore. 
Debnath, A.K. and Chin, H.C. (2006). Analysis of Marine Conflicts. Proceedings of the Nineteenth KKCNN Symposium on Civil Engineering, Kyoto, Japan.

Debnath, A.K. and H.C. Chin (2007). Analysis of Involved Parties in Port Water Conflicts. Proceedings of the Twentieth KKCNN Symposium on Civil Engineering, Jeju, Korea.

Debnath, A.K. and Chin, H.C. (2009a). Hierarchical Modeling of Perceived Collision Risks in Port Fairways. Transportation Research Record: Journal of the Transportation Research Board, 2100, 68-75.

Debnath, A.K. and Chin, H.C. (2009b). Perceived Collision Risks in Anchorages: A Hierarchical Ordered Probit Analysis. Proceedings of the The 8th EASTS Conference, Surabaya, Indonesia.

Debnath, A.K. and Chin, H.C. (2010). Navigational Traffic Conflict Technique: A Proactive Approach to Quantitative Measurement of Collision Risks in Port Waters. The Journal of Navigation, 63(1), 137-152.

Debnath, A.K., Chin, H.C. and Haque, M. M. (2011). Modelling port water collision risk using traffic conflicts. The Journal of Navigation, 64(4), 645-655.

Goerlandt, F. and Kujala, P. (2014). On the reliability and validity of ship-ship collision risk analysis in light of different perspectives on risk. Safety Science, 62(0), 348-365.

Goerlandt, F., K. Ståhlberg and Kujala, P. (2012). "Influence of impact scenario models on collision risk analysis." Ocean Engineering 47(0), 74-87.

Goossens, L.H.J. and Glansdorp, C.C. (1998). Operational Benefits and Risk Reduction of Marine Accidents. The Journal of Navigation, 51(3), 368-381.

Hänninen, M., Mazaheri, A., Kujala, P., Montewka, J., Laaksonen, P., Salmiovirta, M. and Klang, M. (2013). Expert elicitation of a navigation service implementation effects on ship groundings and collisions in the Gulf of Finland. Proceedings of the Institution of Mechanical Engineers, Part O: Journal of Risk and Reliability.

IALA. (1980). IALA Maritime Buoyage System, International Association of Marine Aids to Navigation and Lighthouse Authorities, France.

IALA. (2012). IWRAP MKII Wiki. International Association of Marine Aids to Navigation and Lighthouse Authorities.

Li, Q., Lam, J. and Fan, H. (2014). Multi-link-ahead Conflicts Prediction in Dynamic Seaport Environments. Simulations, Serious Games and Their Applications. Y. Cai and S. L. Goei, Springer Singapore, 69-84.

Liu, C.-P., Liang, G.-S., Su, Y. and Chu, C.-W. (2006). Navigation Safety Analysis in Taiwanese Ports. The Journal of Navigation, 59(2), 201-211.

Montewka, J., Ehlers, S., Goerlandt, F., Hinz, T., Tabri, K. and Kujala, P. (2014). A framework for risk assessment for maritime transportation systems - A case study for open sea collisions involving RoPax vessels. Reliability Engineering \& System Safety, 124(0), 142-157.

Montewka, J., Goerlandt, F. and Kujala, P. (2012). Determination of collision criteria and causation factors appropriate to a model for estimating the probability of maritime accidents. Ocean Engineering, $\mathbf{4 0}(0)$, 50-61.

Montewka, J., Hinz, T., Kujala, P. and Matusiak, J. (2010). Probability modelling of vessel collisions. Reliability Engineering \& System Safety, 95(5), 573-589.

Qu, X., Meng, Q. and Suyi, L. (2011). Ship collision risk assessment for the Singapore Strait. Accident Analysis \& Prevention, 43(6), 2030-2036.

Silveira, P.A.M., Teixeira, A.P. and Guedes Soares, C. (2013). Use of AIS Data to Characterise Marine Traffic Patterns and Ship Collision Risk off the Coast of Portugal. The Journal of Navigation, 66(6), 879-898.

Guedes Soares, C. and Teixeira, A.P. (2001). Risk assessment in maritime transportation. Reliability Engineering \& System Safety, 74(3), 299-309.

SOLAS. (1974). International Convention for the Safety of Life at Sea 1974. I. M. Organization. London, UK. Chapter V - Regulation 23.

Sormunen, O.-V.E., Goerlandt, F., Häkkinen, J., Posti, A., Hänninen, M., Montewka, J., Ståhlberg, K. and Kujala, P. (2014). Uncertainty in maritime risk analysis: Extended case study on chemical tanker collisions. Proceedings of the Institution of Mechanical Engineers, Part M: Journal of Engineering for the Maritime Environment.

Suman, S., Nagarajan, V., Sha, O.P., Khanfir, S., Kobayashi, E. and Malik, A.M.B.A. (2012). Ship Collision Risk Assessment using AIS Data. International Journal of Innovative Research \& Development, 1(10), 509-524.

Usui, H. (2002). "Navigating Between Anchored Ships and Manoeuvering Difficulty." The Journal of Navigation, 55(2), 277-291. 
van Dorp, J.R. and Merrick, J.W. (2011). On a risk management analysis of oil spill risk using maritime transportation system simulation. Annals of Operations Research, 187(1), 249-277.

Weng, J., Meng, Q. and Qu, X. (2012). Vessel collision frequency estimation in the Singapore Strait. Journal of Navigation, 65, 207-221.

Yip, T.L. (2008). Port traffic risks - A study of accidents in Hong Kong waters. Transportation Research Part E: Logistics and Transportation Review, 44(5), 921-931.

Zaman, M.B., Kobayashi, E., Wakabayashi, N., Khanfir, S., Pitana, T. and Maimun, A. (2014). Fuzzy FMEA model for risk evaluation of ship collisions in the Malacca Strait: based on AIS data. Journal of Simulation, 8, 91-104.

Zhang, P. and Zhao, J. (2013). The Obligations of an Anchored Vessel to Avoid Collision at Sea. The Journal of Navigation, 66(3), 473-477. 